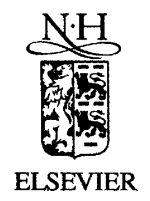

Information Sciences 148 (2002) 139-154

INFORMATION

SCIENCES

AN INIERNATIONAL JOURNAL

www.elsevier.com/locate/ins

\title{
Diagonalisation of a class of multivariable system via an actuator linearisation technique
}

\author{
J.V. Ringwood * \\ Department of Electronic Engineering, National University of Ireland, Maynooth, County Kildare, \\ Ireland
}

Received 11 November 2000; received in revised form 3 January 2002; accepted 13 April 2002

\begin{abstract}
Many multivariable (systems with many inputs/outputs) industrial processes can, to a good degree of approximation, be modelled by a transfer function matrix, where all of the interaction occurs in a matrix of constant coefficients. This reflects the fact that the dynamics of the section in which the interaction occurs are very fast compared with the other dynamics in the system. Examples of such systems include steel rolling mills and boiler systems.

Such multivariable systems are relatively easy to design controllers for, since the system may be diagonalised by an inverse of the constant gain matrix, followed by suitable single-loop dynamic compensation. However, this approach depends on the linearity of the dynamical elements in the system. Such a condition is voilated by the presence of non-linear actuators, which are a feature of many industrial systems. The presence of such actuators within a multivariable control system as described above can cause very significant interaction problems, with associated degradation in performance, particularly during transients.

This paper describes a straightforward technique, which is effective in linearising typical non-linear industrial actuators, allowing diagonalisation to be effectively achieved at all frequencies. The technique relies on a simple describing function analysis and manifests itself as a time-varying linearising precompensator for each non-linear actuator. A simple example is used to demonstrate the effectiveness of the method and it is then shown in application with multivariable boiler and steel mill models.

(c) 2002 Elsevier Science Inc. All rights reserved.
\end{abstract}

\footnotetext{
*Fax: +353-1-708-6027.

E-mail address: john.ringwood@eeng.may.ie (J.V. Ringwood).
} 
Keywords: Automation; Multivariable systems; Actuator linearisation; Steel mills; Boiler control

\section{Introduction}

Many techniques exist for the analysis of single-input, single-output (SISO) systems. The range of techniques for multivariable systems (systems with many inputs and/or outputs), however, is rather limited [1], One solution to a multivariable problem is to ignore the interaction between paths through the system and try to relate each system output to a particular input, followed by a number of single loop designs. Such an approach may be successful where the degree of interaction between paths is low, but may fail in the majority of cases. A more sophisticated approach is to apply a diagonalising precompensator followed by a number of single loop designs. Such an approach is adopted in a number of multivariable control design procedures [1], and has the appeal that any number of SISO design techniques may be applied once diagonalisation is effected. However, the diagonalisation itself is not straightforward, particularly if the system contains significantly different dynamics in each path or nonlinearities. This paper addresses classes of systems where the interaction occurs in a matrix of constant gains but with differing non-linear actuator dynamics in each path.

There is a wealth of literature on actuator linearisation. Many studies use feedback linearisation, which uses an inverse actuator model and relies on the sensitivity reduction of the feedback to compensate for any residual non-linearity, e.g., [2]. However, this cannot be applied to relay-type systems, since the relay represents a non-invertible characteristic. A number of studies look at small signal linearised representations, e.g., [3], but these are only valid for a very small range of operation. Most studies treat saturating actuators, e.g., [4,5], but this considers an essentially linear actuator, which has an amplitude limitation. Finally, [6] considers an electro servo-hydraulic valve, but linearises the static characteristic. The approach in this paper is to deal with the complete actuator servo loop and to develop a compensation method, which achieves something close to exact linearisation of the servo loop, over its complete range of operation.

Non-linear actuators of the form shown in Fig. 1 are found in many systems, both hydraulic and electrical. The effect of the relay shown is often manifested as a controller output which has only 'raise' or 'lower' signals, limiting the maximum rate of change of the output, often for safety reasons or due to physical limitations.

Such actuators give a ramp type output, not normally a problem in scalar systems. However, in multivariable systems, these types of actuators can in- 


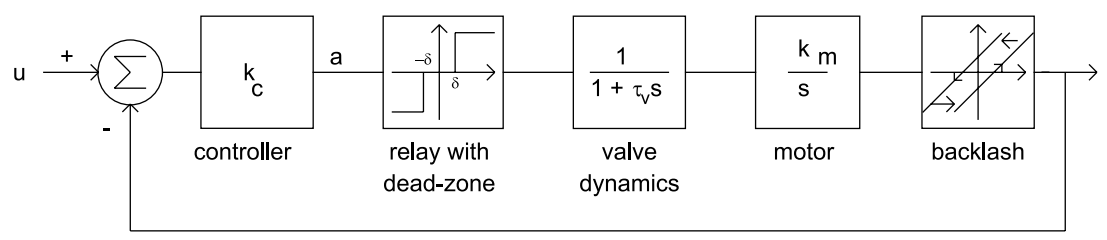

Fig. 1. Actuator block diagram.

troduce severe interaction between the signal paths, when an attempt is made to diagonalise the system. Consider the case of an $n$ by $n$ canonical system, where all of the interaction occurs in a matrix of constant gains, $G_{\mathrm{m}}$, with $g(s)$ representing a linear, scalar dynamic transfer function (possibly including pure delay)

$$
y=g(s) G_{\mathrm{m}}\left[f_{1}\left(u_{1}\right) f_{2}\left(u_{2}\right) \ldots f_{n}\left(u_{n}\right)\right]^{\mathrm{T}},
$$

where

$$
\begin{aligned}
& G_{\mathrm{m}} \in \mathfrak{R}^{n \times n}, \quad g(s) \in \mathfrak{R}(s), \\
& y \in \mathfrak{R}^{n}, \quad u_{1}, u_{2}, \ldots, u_{n} \in \mathfrak{R} .
\end{aligned}
$$

The actuators are represented by the non-linear functions, $f_{\mathrm{i}}\left(u_{\mathrm{i}}\right)$, with $u_{\mathrm{i}}$ being the respective inputs to these actuators. If compensators $c_{1}(\cdot), c_{2}(\cdot), \ldots, c_{n}(\cdot)$ can be found so that:

$$
f_{1}\left(c_{1}\left(u^{*}\right)\right)=f_{2}\left(c_{2}\left(u^{*}\right)\right)=\cdots=f_{n}\left(c_{n}\left(u^{*}\right)\right)=q(s) u^{*},
$$

where $u^{*}$ is the input to the precompensator, the system in Eq. (1) may be easily diagonalised by the application of $G_{\mathrm{m}}^{-1}$ (where $G_{\mathrm{m}}^{-1}$ exists) as shown in Fig. 2, to give:

$$
y=q(s) g(s) G_{\mathrm{m}} u^{*}=g(s) q(s) I_{n} w,
$$

where

$$
u, u^{*}, w \in \mathfrak{R}^{n}
$$

and single loop compensation may be applied as appropriate to give suitable dynamic performance. See [7] and [8] respectively for solutions where $G_{\mathrm{m}}$ is singular or non-square.

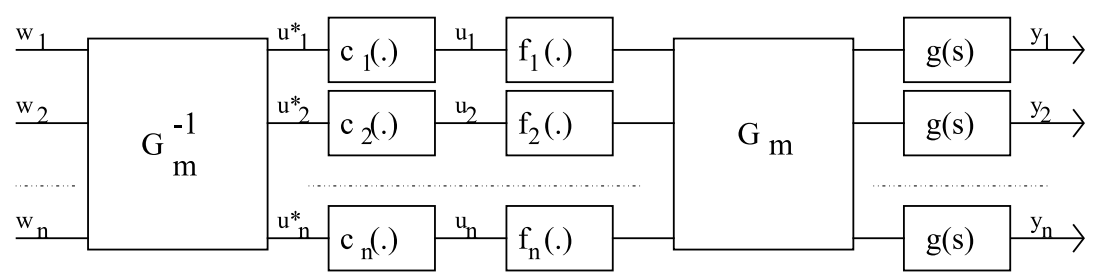

Fig. 2. Diagonalisation of non-linear system. 
Section 2 demonstrates a simple method for the determination of a compensator set $c_{1}(\cdot), c_{2}(\cdot), \ldots, c_{n}(\cdot)$ which linearises the actuators, with a $2 \times 2$ example provided in Section 3, indicating the decoupling performance of the technique. The application of the technique to steel mill and boiler systems is shown in Sections 4 and 5 respectively, and conclusions are drawn in Section 6.

\section{Actuator linearisation}

The actuator system under examination is shown in Fig. 1. The dynamics of the hydraulic valve (not present for an electrical actuator) are normally assumed to be considerably faster than the motor. The backlash on the position output is due to gearing or a rack and pinion arrangement on the actuator output. To prevent hunting (small oscillation limit cycling) due to the presence of backlash [9], a small amount of dead-zone is usually introduced into the relay. For analysis purposes, the dominant components of the actuator system are taken to be the controller gain, relay and motor. It will be shown that the omission of the backlash, dead-zone and valve dynamics from the model will not result in any significant errors, while maintaining computational simplicity in the linearising compensator.

Obtaining the describing function for the relay gives the well-known result [10]:

$$
N_{r}(a)=4 /(\pi a)
$$

where $a$ is the amplitude of the signal entering the relay. The extension for a relay with dead zone is [9]:

$$
N_{r d z}(a)=\frac{4 \delta}{\pi a} \sqrt{1-\left(\frac{\delta}{2 a}\right)},
$$

where $\delta$ is the amount of dead zone. Evaluating the closed loop transfer function for the actuator system of Fig. 1, using the describing function approximation of Eq. (2) for the relay, yields:

$$
T(s, a)=\frac{1}{1+\left(\pi a / 4 k_{\mathrm{c}} k_{\mathrm{m}}\right) s},
$$

where $k_{\mathrm{c}}$ and $k_{\mathrm{m}}$ represent the gains of the controller and motor respectively. Eq. (3) represents a first order system with an amplitude dependent (equivalent) time constant of:

$$
\tau_{\mathrm{e}}=\frac{\pi a}{4 k_{\mathrm{c}} k_{\mathrm{m}}} .
$$


If a compensator, $c(\cdot)$, is now placed in cascade with the actuator, where

$$
c(s, a)=\frac{1+\tau_{\mathrm{e}}(a) s}{1+\tau_{n} s}
$$

then the cascade combination of the compensator and actuator reduces to a first order LTI system with time constant, $\tau_{n}$, which may be chosen by the designer subject to the obvious limitation that the compensated system cannot move faster than the rate limit imposed by $k_{\mathrm{m}}$. Note that the signal input to the relay characteristic is normally available as an electrical signal. As an illustration of the performance of such a compensator, consider the following two actuator systems:

\begin{tabular}{llllll}
\hline & $\begin{array}{l}\text { Cont. } \\
\text { gain }\left(k_{\mathrm{c}}\right)\end{array}$ & $\begin{array}{l}\text { Motor } \\
\text { gain }\left(k_{\mathrm{m}}\right)\end{array}$ & $\begin{array}{l}\text { Dead-zone } \\
(\delta)\end{array}$ & $\begin{array}{l}\text { Backlash } \\
(\beta)\end{array}$ & $\begin{array}{l}\text { Time } \\
\text { const. }\left(\tau_{v}\right)\end{array}$ \\
\hline Actuator A & 0.7 & 3.14 & 0.25 & 0.5 & 0.1 \\
Actuator B & 0.3 & 8.0 & 0.25 & 0.3 & 0.05 \\
\hline
\end{tabular}

Fig. 3 shows the step response of both actuators with their associated precompensators along with the step response of an LTI first order system with a

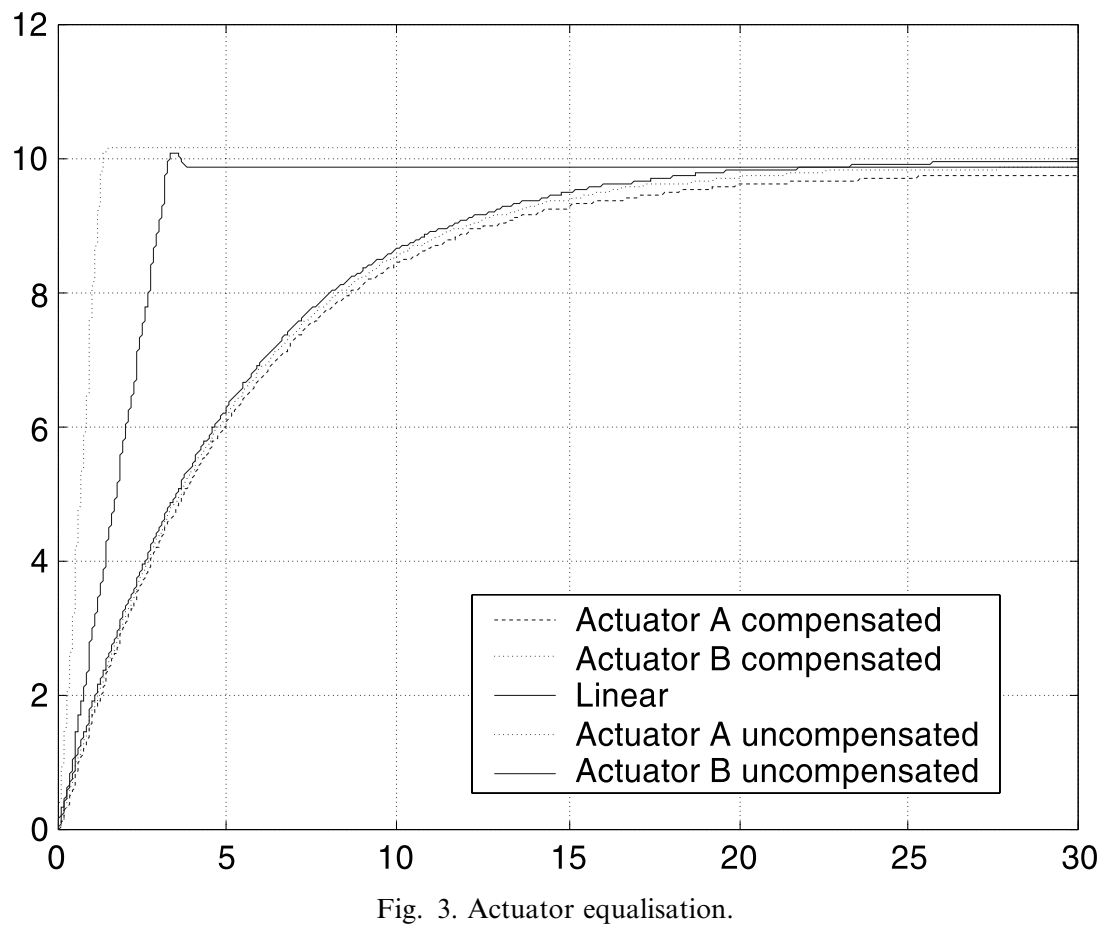



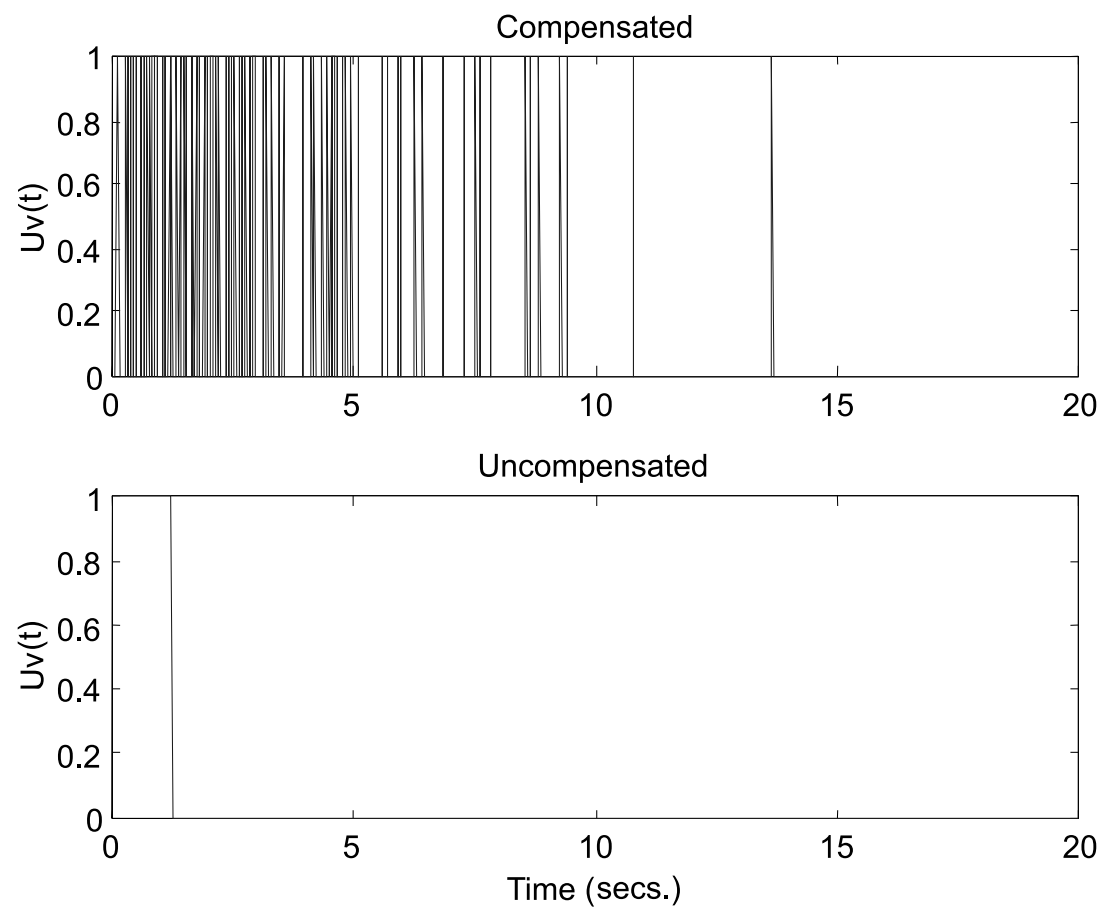

Fig. 4. Control signals to hydraulic valve for compensated (upper) and uncompensated (lower) actuators.

time constant of $5 \mathrm{~s}$, since $\tau_{n}$ was chosen to be $5 \mathrm{~s}$. Fig. 4 shows the control signals for compensated and uncompensated actuators.

\section{A diagonalisation example}

A $2 \times 2$ example will be taken here to illustrate the use of the linearising precompensator in the diagonalisation as shown in Section 1. The actuator parameters are those given in the table in Section 2. The scalar dynamics, $g(s)$, will be set to unity, facilitating clearer observation of the output responses. For this example, $\tau_{n}$ was chosen to be $3 \mathrm{~s}$, with

$$
G_{\mathrm{m}}=\left[\begin{array}{ll}
2 & 1 \\
3 & 3
\end{array}\right] \text {, giving } G_{\mathrm{m}}^{-1}=\left[\begin{array}{cc}
1 & -\frac{1}{3} \\
-1 & \frac{2}{3}
\end{array}\right] .
$$

Fig. 5 shows the responses of the two outputs with a step in path 1 and a zero input on the other for the uncompensated non-linear system. Fig. 6 shows 


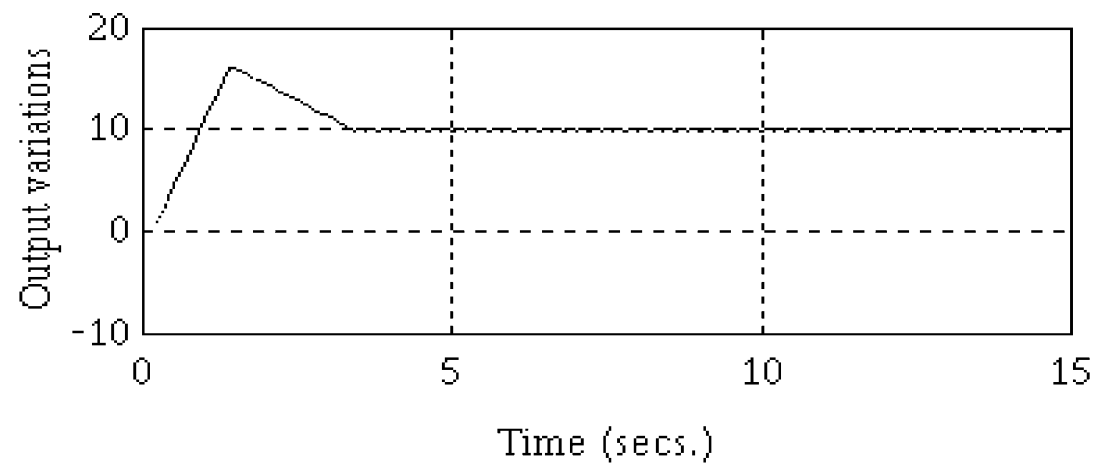

Fig. 5. Multivariable response for uncompensated system.

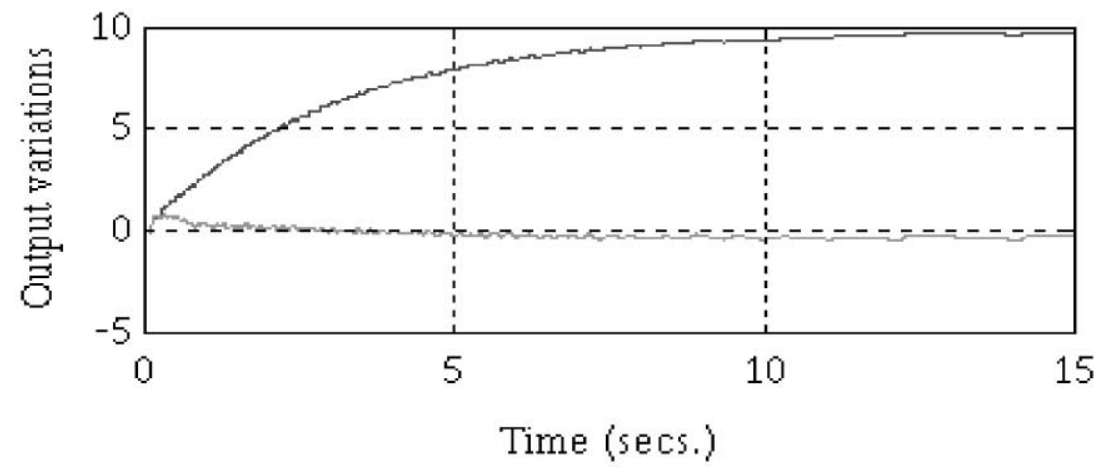

Fig. 6. Multivariable response for compensated system.

the corresponding response for the compensated system. Although the uncompensated system is diagonal at zero frequency (steady-state response), significant interactions occur in the transient response. In certain applications, this may be unacceptable, with the possibility of large undesirable excursions in the outputs leading to critical operating conditions, for example tearing of the strip in steel processing.

\section{Boiler system application}

The primary purpose of an industrial boiler is to produce steam, given fuel, air and feedwater. This study concentrates on the combustion side of the problem, where fuel and air are combined in a combustion chamber to give heat energy [11]. An important by-product of the combustion process is the 


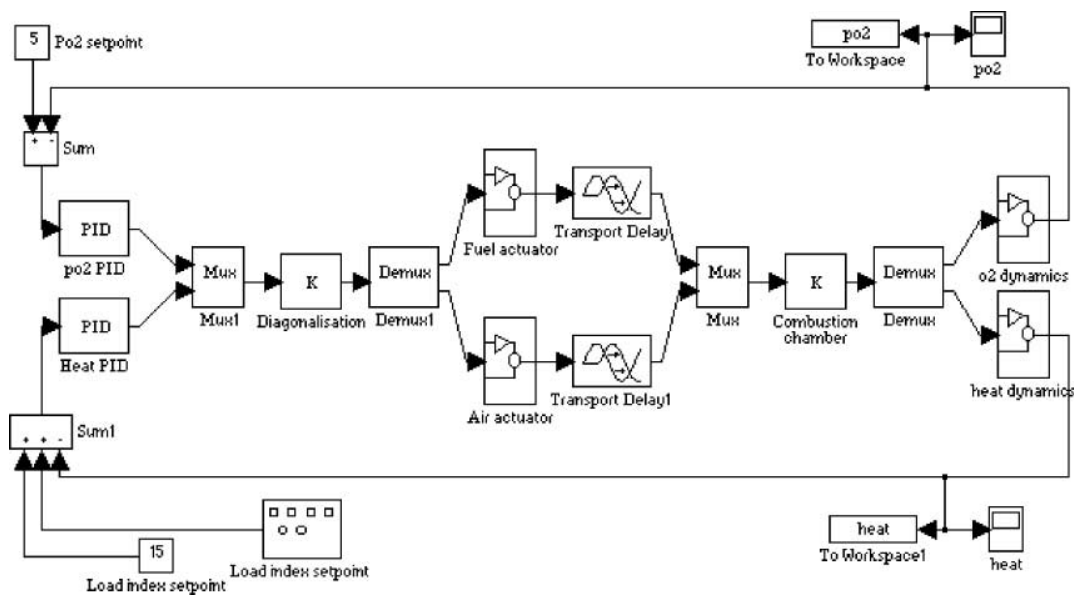

Fig. 7. Boiler model and control system.

gas, which is discharged in the flue. The constituents of this gas must be carefully controlled for environmental reasons. The mixing of the fuel and air determines both the heat generated and the gas composition in the flue. From an energy point of view, only the exact amount of air required to burn the fuel should be supplied (stochiometric ratio). Insufficient air means that not all the fuel is burned and excess air propagates through the combustion chamber, drawing heat from the system. Although the stochiometric ratio is desired from an energy point of view, it is desirable to maintain the $\% \mathrm{O}_{2}$ in the stack gasses at about $1-10 \%$ for environmental reasons. Supplying a quantity of air below the stochiometric point results in the production of black smoke, which is prohibited by strong environmental regulations.

The problem is a multivariable one, with both the heat output and $\% \mathrm{O}_{2}$ depending on fuel and air flow rates. The control philosophy for the boiler subsystem is shown in Fig. 7. Note that the model structure accords with that given in Fig. 2. The interaction between paths takes place in the combustion chamber, with linearised constant gains representing the combustion chamber effects, since the dynamics of the combustion chamber are very fast compared to the actuator, gas probe and heat propagation dynamics.

The interaction matrix in the boiler is given by

$$
G_{\mathrm{m}}=\left[\begin{array}{cc}
8 & 5 \\
-2 & 3
\end{array}\right] \text {. }
$$

Transport delays are incurred in both the air and fuel paths before the combustion chamber and the dynamics after the combustion chamber also contain delays, along with first order lag terms. The actuators are as in Fig. 1, with the following parameters: 


\begin{tabular}{llllll}
\hline & $\begin{array}{l}\text { Cont. } \\
\text { gain }\left(k_{\mathrm{c}}\right)\end{array}$ & $\begin{array}{l}\text { Motor } \\
\text { gain }\left(k_{\mathrm{m}}\right)\end{array}$ & $\begin{array}{l}\text { Dead-zone } \\
(\delta)\end{array}$ & $\begin{array}{l}\text { Backlash } \\
(\beta)\end{array}$ & $\begin{array}{l}\text { Time } \\
\text { const. }\left(\tau_{v}\right)\end{array}$ \\
\hline Air actuator & 0.5 & 1.1 & 0.01 & 0.0 & 0.1 \\
Fuel actuator & 0.3 & 8.0 & 0.01 & 0.0 & 0.05 \\
\hline
\end{tabular}

The controller for the boiler subsystem contains a diagonalisation block, which is the inverse of the matrix in (7), with two PI controllers used for the dynamic compensation of each path. The controller parameters are:

\begin{tabular}{lll}
\hline Controller parameters & Proportional gain & Integral gain \\
\hline Heat controller & 0.6 & 0.35 \\
$\% \mathrm{O}_{2}$ controller & 0.8 & 0.45 \\
\hline
\end{tabular}

The model is normalised for $0-100 \%$ load index as a heat setpoint, with the $\% \mathrm{O}_{2}$ setpoint set at a nominal value of $5 \%$. To examine the dynamics of the system, the load index is varied cyclically between 10-20\%. Load index variations are caused by increases or decreases in steam demand, which manifest themselves as decreases or increases in steam pressure in the steam drum (or

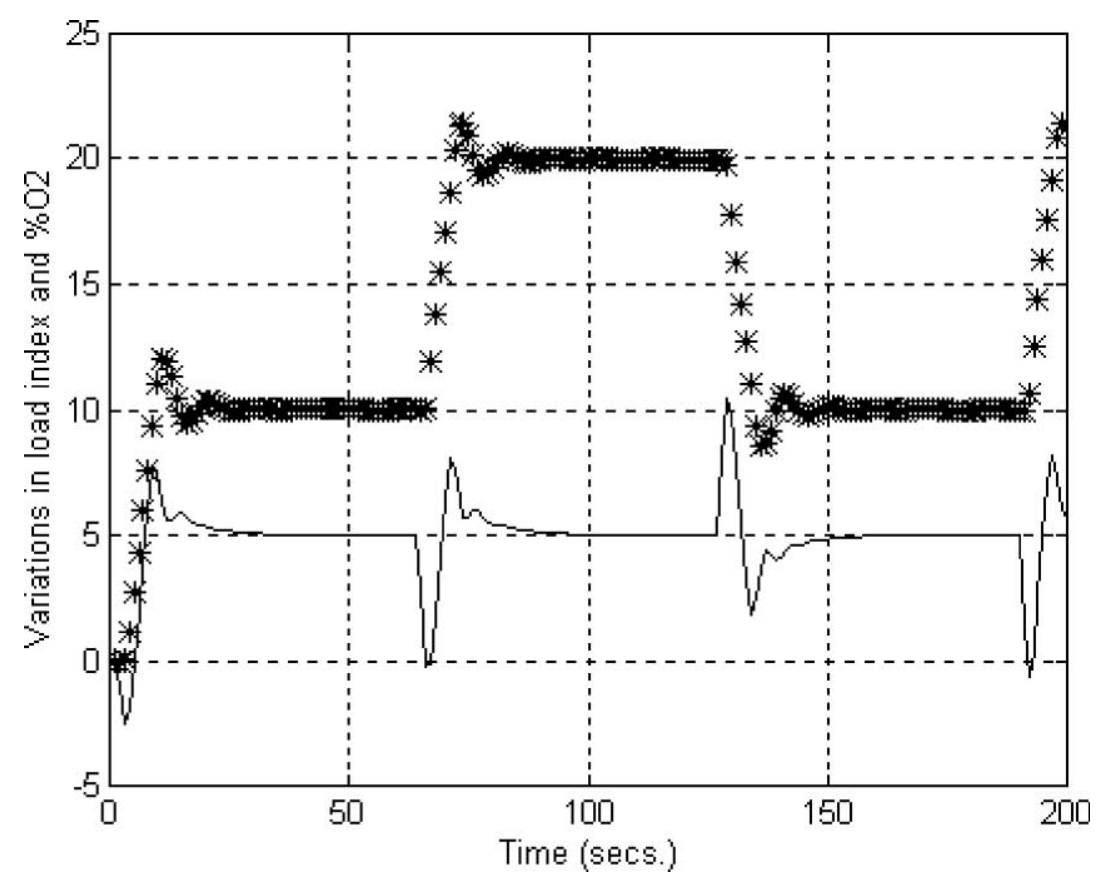

Fig. 8. Response with no actuator precomposition. 


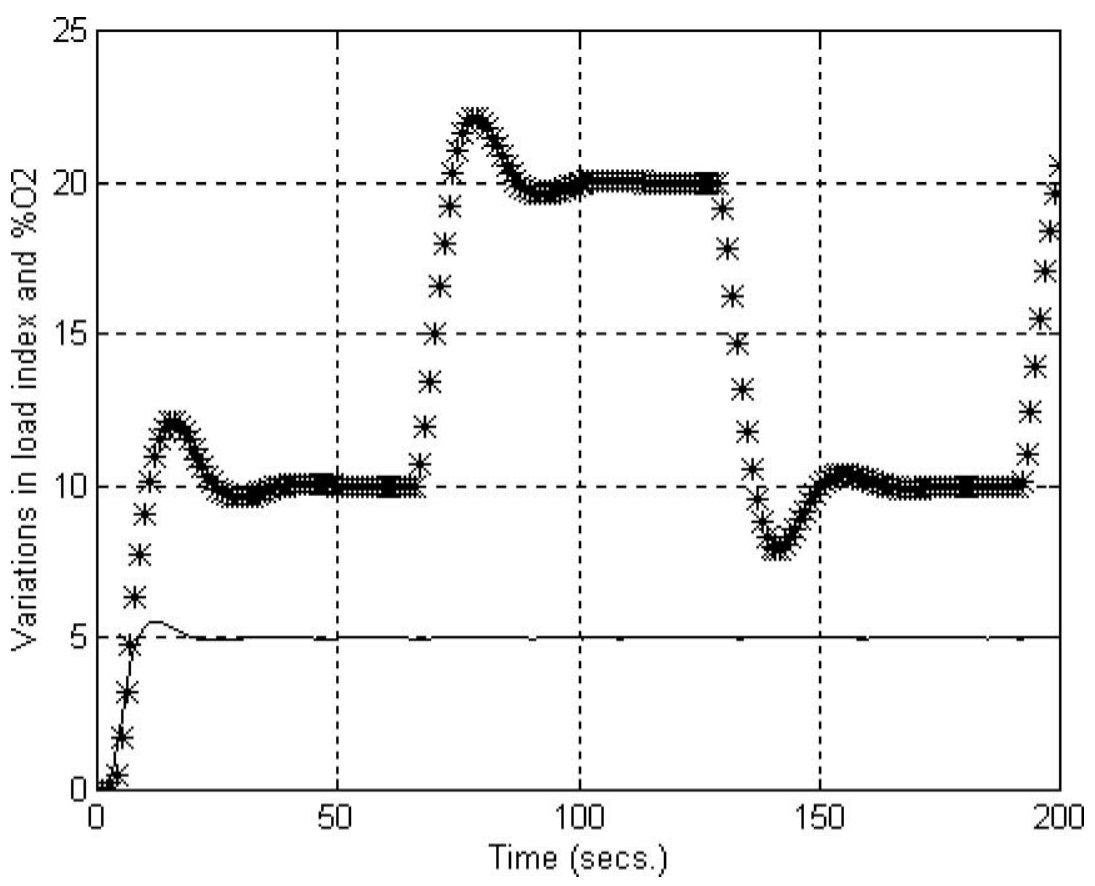

Fig. 9. Response with actuator precomposition.

superheater, where present) respectively. To maintain steam production consistent with the load the load index should be increased or decreased respectively. Fig. 8 demonstrates the variations in load index (indicated by *) and $\% \mathrm{O}_{2}$ (solid line) where no actuator precompensation is used.

Note from Fig. 8 that considerable interaction occurs between the two controlled variables, the variations in load index (which are normally frequent) causing considerable excursions in the $\% \mathrm{O}_{2}$. Indeed, in spite of the fact that the $\% \mathrm{O}_{2}$ setpoint is at $5 \%$, the actual $\% \mathrm{O}_{2}$ in the stack drops below $0 \%$, indicating black smoke production. Fig. 9 shows the situation with actuator precompensators, as discussed in Section 2, in place.

Note that true diagonalisation has been achieved, at the expense of some speed in the time response. However, the regulation of the $\% \mathrm{O}_{2}$ is good, allowing the $\% \mathrm{O}_{2}$ setpoint to be lowered, if desired, for more efficient boiler operation, without the risk of black smoke production (Fig. 9).

\section{Steel mill application}

A schematic of the steel mill system, including the controller, is shown in Fig. 10 [8]. The mill is used to roll stainless steel with the objective of reducing 
the strip gauge. During this rolling process, undesirable stresses can be set up in the strip (sometimes termed "bad shape") and the mill subsystem shown in Fig. 10 is there to attempt to ensure that a uniform, or zero, stress profile across the strip width is obtained and corresponds to the shape control subsystem. In extreme cases, internal strip stress can cause tearing of the strip. Shape is controlled via modifications of the roll-gap profile.

The shape control subsystem consists of two mechanisms; the As-U-Rolls (AUR's), which effectively create point loads at evenly spaced intervals across the rolling cluster, and the First Intermediate Rolls (FIR's) which are tapered and can affect the roll-gap profile by their lateral movement in or out of the rolling cluster. The hydraulic actuators which drive each of these mechanisms are of the form shown in Fig. 1 and operate at different speeds, with parameters:

\begin{tabular}{llllll}
\hline & $\begin{array}{l}\text { Cont. } \\
\text { gain }\left(k_{\mathrm{c}}\right)\end{array}$ & $\begin{array}{l}\text { Motor } \\
\text { gain }\left(k_{\mathrm{m}}\right)\end{array}$ & $\begin{array}{l}\text { Dead-zone } \\
(\delta)\end{array}$ & $\begin{array}{l}\text { Backlash } \\
(\beta)\end{array}$ & $\begin{array}{l}\text { Time } \\
\text { const. }\left(\tau_{v}\right)\end{array}$ \\
\hline Actuator A & 0.7 & 3.14 & 0.25 & 0.5 & 0.1 \\
Actuator B & 0.3 & 8.0 & 0.25 & 0.3 & 0.05 \\
\hline
\end{tabular}

As in (1), the mill model is of the form:

$$
\begin{aligned}
& y=h(s)\left[G_{a} f_{a}\left(u_{a}\right) \quad G_{\mathrm{i}} f_{\mathrm{i}}\left(u_{\mathrm{i}}\right)\right], \\
& G_{a} \in \mathfrak{R}^{8 \times 8}, \quad G_{\mathrm{i}} \in \mathfrak{R}^{8 \times 2},
\end{aligned}
$$

where the $i$ and $a$ subscripts denote the FIR's and AUR's respectively. Given that a set of linearising actuator precompensators may be found, as discussed in Section 2, the matrix which must be diagonalised is

$$
G_{\mathrm{m}}=\left[\begin{array}{ll}
G_{\mathrm{a}} & G_{\mathrm{i}}
\end{array}\right] \text {. }
$$

However, it transpires that this matrix is both non-square and non-full-rank. A pseudo-diagonalisation is achieved using the singular value decomposition [12]

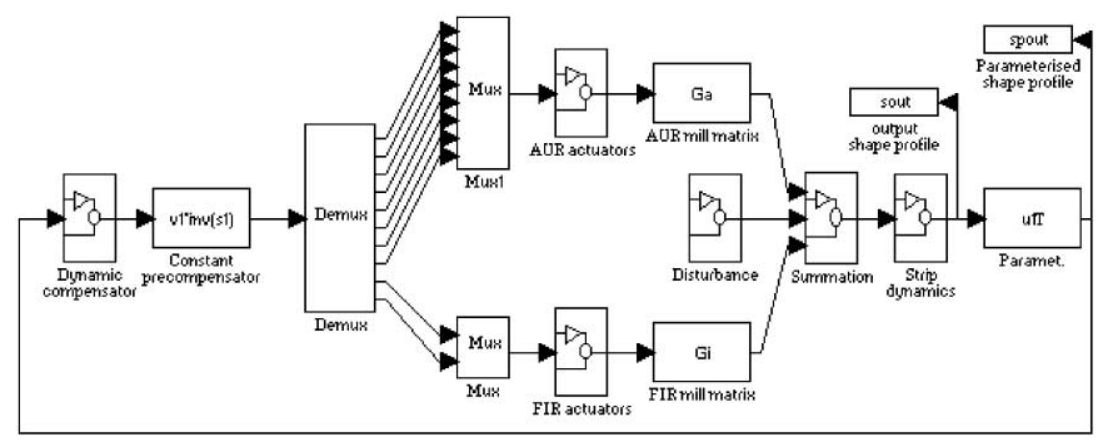

Fig. 10. Mill model with shape control system. 

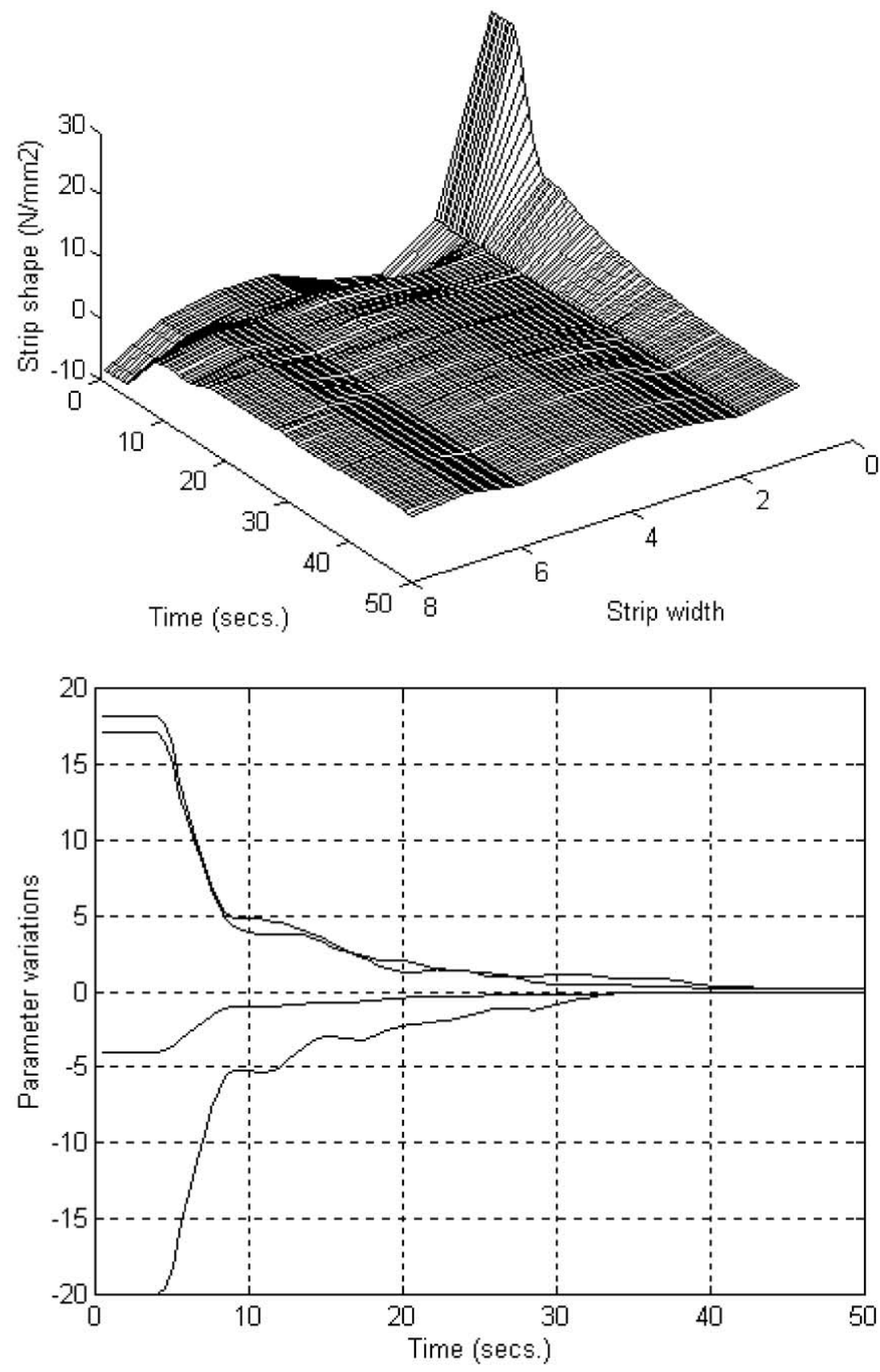

Fig. 11. Shape profile and parametric variations (actuator uncompensated). 

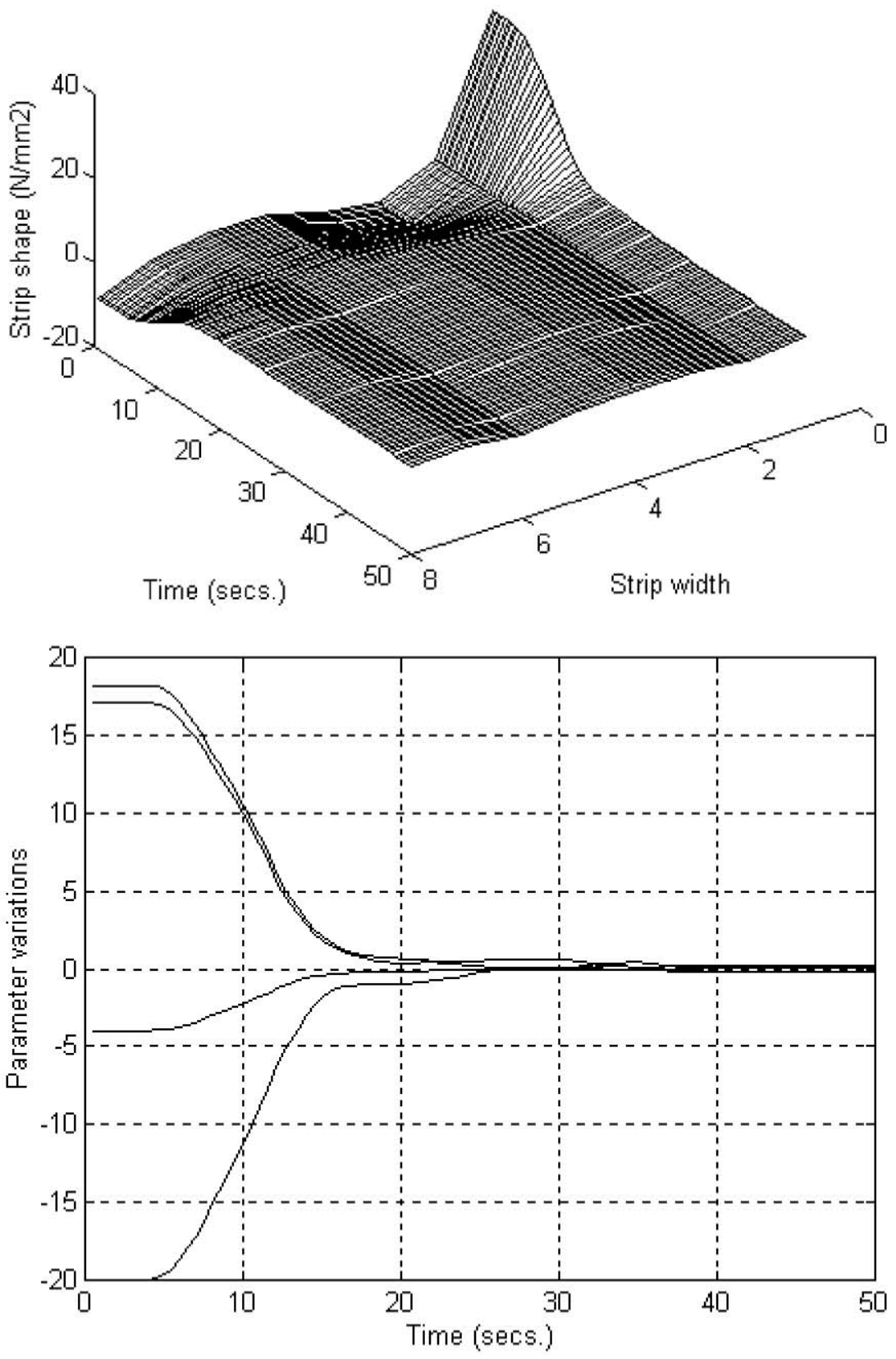

Fig. 12. Shape profile and parametric variations (actuator compensated). 
which diagonalises the system with respect to the four principal singular values. Given that $G_{\mathrm{m}}$ is decomposed as

$$
\begin{aligned}
& G_{\mathrm{m}}=\left[\begin{array}{ll}
U_{1} & U_{2}
\end{array}\right]\left[\begin{array}{ccc}
\Sigma_{1} & 0 & 0 \\
0 & \Sigma_{2} & 0
\end{array}\right]\left[\begin{array}{c}
V_{1}^{\mathrm{T}} \\
V_{2}^{\mathrm{T}}
\end{array}\right] \\
& U_{1}, U_{2} \in \mathfrak{R}^{8 \times 4}, \quad V_{1} \in \mathfrak{R}^{10 \times 4}, \quad V_{2} \in \mathfrak{R}^{10 \times 6}, \quad \Sigma_{1}, \Sigma_{2} \in \mathfrak{R}^{4 \times 4}
\end{aligned}
$$

the required pseudo-diagonalisation is achieved by the choice of compensator as

$$
K=V_{1} \Sigma_{1}^{-1} U_{1}^{\mathrm{T}} .
$$

Further details and properties of this diagonalisation are provided in [8]. One important point worth noting is that is that the singular vectors correspond to polynomial profiles; thus the first singular vector accords with a linear stress profile, the second quadratic, and so on. Looking at the parametric shape profile obtained after postmultiplying the shape profile with $U_{1}^{\mathrm{T}}$ gives the coefficients of first to fourth order stress profiles in the strip. Fig. 10 shows a schematic of the system with the controller.

The disturbance represents the incoming strip shape profile. The desired profile is uniformly flat i.e. the first four coefficients of the parametric profile should ideally be zero. Fig. 11 shows the variations in the shape and parametric shape profiles respectively for a system with no actuator compensation. Note that control is applied after $3 \mathrm{~s}$.

Note the sharp contours in Fig. 11 (upper) and the highly non-linear response in Fig. 11 (lower) resulting from uneven actuator action. Fig. 12 demonstrates the variations in the shape and parametric shape profiles for a system with actuator compensation.

Note the smoother shape profile response from Fig. 12. This is important, since sharp variations in the profile can result in tearing of the steel strip. Interestingly, it can be seen that although the actuator precompensation technique tends to deteriorate the transient response for individual actuators, the overall settling time for the parametric variations has been considerably improved.

\section{Conclusions}

The paper presents a simple but effective technique for actuator linearisation. The price paid for achieving a smooth linear response is degradation in the rise time and some extra activity in the signal to the servo valve, but this may be acceptable when considered with possibly large undesirable excursions in certain outputs for an uncompensated multivariable system. The diagonalisation technique is applicable to systems where the interaction between 
multivariable channels occurs in a matrix of constant coefficients. The remaining dynamical (scalar) components must be linear, but may contain pure time delay. For the actuator configuration shown in Fig. 1, the describing function provides a good representation, since output harmonics are attenuated heavily by the motor dynamics. This is evidenced by the linearisation example in Section 2 and by frequency response measures, which have also been performed. The results of Sections 2 and 3 also validate the approximations made in neglecting the effects of backlash, dead-zone and valve dynamics in the construction of the linearising precompensator. Further computational simplicity is achieved if the compensators are realised in state-space controllable form, with the result that the discrete time A and B matrices are fixed, the amplitude-dependent terms entering only into the $\mathrm{C}$ and $\mathrm{D}$ matrices, which remain invariant under the transformation from continuous to discrete time. Finally, some comment is merited regarding the robustness of the precompensators with respect to uncertainty in the actuator parameters, such as motor gain, etc. In this respect, the scheme employed is of the general form employed in internal model control, applicable to both linear and non-linear systems [13], where an inverse feedforward model is employed, but an overall feedback with dynamic compensator is also used. This structure is clearly evident in both Figs. 7 and 10.

\section{References}

[1] D.J. Bell, P.A. Cook, N. Munro (Eds.), Design of Modern Control Systems, Peter Peregrinus, 1982.

[2] X. Ma, G. Tao, Adaptive actuator compensation control with feedback linearization, IEEE Trans. Autom. Cont. 45 (9) (2000) 1705-1710.

[3] X. Lin, S. Scavarda, Linearized analysis of an electropneumatic servovalve/long pneumatic actuator assembly using bond graph, in: Proc. Int. Conf. Syst., Man Cybern., vol. 1, 1993, pp. $555-560$.

[4] J. Grantz, S. Valluri, M. Soroush, Discrete-time nonlinear control of processes with actuator saturation, AIChE J. 44 (7) (1998) 1701-1705.

[5] C. Gokcek, P.T. Kabamba, S.M. Meerkov, An LQR/LQG theory for systems with saturating actuators, IEEE Trans. Autom. Cont. 46 (10) (2001) 1529-1542.

[6] H. Hahn, A. Piepenbrink, K.-D. Leimbach, Input/output linearization control of an electro servo-hydraulic actuator, in: Proc. 3rd IEEE Conf. Control Appl., vol. 2, 1994, pp. 995-1000.

[7] J.V. Ringwood, D.H. Owens, M.J. Grimble, Feedback design of a canonical multivariable system with application to shape control in a Sendzimir mill, Trans. ASME, J. Dyn. Syst., Meas., Control March (1994).

[8] J.V. Ringwood, M.J. Grimble, Shape control in sendzimir mills using both crown and intermediate roll actuators, IEEE Trans. Autom. Cont. AC-35 (4) (1990).

[9] D.P. Atherton, Nonlinear Control Engineering, Van Nostrand Reinhold, New York, 1982.

[10] I.J. Nagrath, N. Gopal, Control Systems Engineering, Wiley Eastern, New York, 1982.

[11] B. Molloy, Modelling and predictive control of a drum-type boiler, Ph.D. thesis, Dublin City University, 1997. 
[12] V.C. Klema, A.J. Laub, The singular value decomposition: its computation and some applications, IEEE Trans. Autom. Control AC-25 (2) (1980).

[13] J. Guan, D.H. Owens, Optimal mode choice and robustness optimization in the internal model control scheme with parameter uncertainty plants, in: Proc. Control 88, UK, 1988, pp. 695700 . 